\title{
Joint Optimization for Integrated Wireless and Wireline Networks
}

\author{
Seong-Lyong Gong, Hee-Tae Roh, Jang-Won Lee \\ Dept. of Electrical \& Electronic Engineering, \\ Yonsei University, Seoul, Korea \\ Email: \{slgong, heetae-roh, jangwon\}@yonsei.ac.kr
}

\author{
Myung Kil Ahn, Byoung In Cho \\ Agency for Defense Development (ADD), \\ Seoul, Korea \\ Email: \{happymkahn, fifth $\} @$ add.re.kr
}

\begin{abstract}
In this paper, we study a cross-layer optimization problem for a network that consists of one wireline core network and multiple wireless access networks. We consider four layers among five layers in the network layering architecture: application characteristics through the utility function and quality of service requirements in the application layer; end-to-end flow control in the transport layer; opportunistic scheduling in the data link layer; adaptive modulation and coding in the physical layer. We formulate a stochastic optimization problem considering above four layers and both wireline and wireless parts of the network jointly that results in a utility-based joint endto-end flow control and opportunistic scheduling problem for the integrated wireless and wireline network. We solve the problem by using a dual approach and a stochastic sub-gradient algorithm. The developed algorithm can be implemented in a distributed way: vertically among four layers and horizontally among all entities in the network, clearly showing what should be done in each layer and each entity and what parameters should be exchanged between layers vertically and between network entities horizontally.
\end{abstract}

\section{INTRODUCTION}

During last two decades, there have been tremendous advances in both wireline and wireless communication networks. Especially, the advances in wireless networks are more dramatic in providing higher capacity and diverse services by adopting new advanced wireless technologies.

In most wireless networks ${ }^{1}$, wireless users are connected to a base-station (BS) (or an access point (AP)) through two wireless links: uplink and downlink. They transmit their data to the BS through its uplink and receive their data from the BS through its downlink. In general, the $\mathrm{BS}$ is connected to the core network $(\mathrm{CN})$, which is a wireline network, through wireline links and forwards data for wireless users in both directions. Hence, in the infrastructure wireless network, only access networks that are directly connected to end users consist of wireless links, while other links in the $\mathrm{CN}$ are wireline links, as in Fig. 1. In other words, only first and/or last hop links are wireless links in the end-to-end path of a communication.

Since only access networks are consists of wireless links, most research efforts have been focused only on the wireless part of the network, i.e., the wireless access

${ }^{1}$ In this paper, we do not consider wireless ad-hoc and mesh networks.

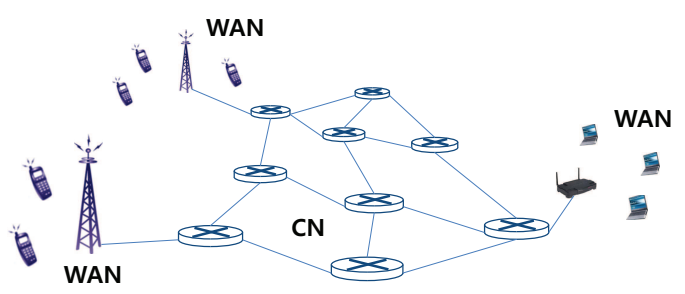

Fig. 1. The integrated wireless and wireline network.

network (WAN), which is only the first or last hop link of the end-to-end communication of wireless users, without considering the entire integrated wireline $\mathrm{CN}$ and WANs. Hence, most researches in wireless networks are focused on protocols only at lower layers such as physical and data link (MAC) layers, and cross-layer optimization of those two layers, ignoring protocols at higher layers such as network and transport layers. In fact, most researches on higher layer issues are done considering only the wireline $\mathrm{CN}$ without considering WANs. However, since the end-to-end communication of a user uses both WANs and wireline $\mathrm{CN}$, not only protocols in lower layers but also protocols in higher layers affect the performance of wireless users. Hence, in wireless networks, cross-layer optimization considering protocols in both higher and lower layers is important to improve system efficiency and satisfactions of users.

In this paper, we study a cross-layer optimization problem for integrated wireline and wireless networks that consists of a wireline $\mathrm{CN}$ and WANs. We consider four layers among five layers in the network layering architecture: application, transport, link (MAC), and physical layers. In the application layer, we consider the satisfaction to the QoS of each session ${ }^{2}$. To this end, in this paper, we will use a concept of utility. Since publications [1], this utility based framework is considered one of the standard frameworks for resource allocation in communication networks. In this approach, each session is assigned a utility function, which represents its degree of satisfaction to the communication

\footnotetext{
2In this paper, we will use terminologies 'user' and 'session' interchangeably.
} 
service according to the amount of resource allocation. By using the appropriate type of utility functions, we can model the characteristics of various network applications.

In the transport layer, we consider end-to-end flow control for each session considering both wireline and wireless links on the end-to-end path of the session. Utility-based flow control has been extensively studied in the literatures, e.g., [1], [2], [3], [4]. However, most of them considered only flow control in wireline CNs.

In MAC and physical layers, we consider opportunistic scheduling of sessions in each WAN. In the MAC layer, in each time-slot, the BS selects a session to which it transmits data through its downlink and a session from which it receives data through its uplink. We consider a system in which an adaptive modulation and coding (AMC) scheme is adopted in the physical layer, i.e., the transmission data rate of a session can be adapted to the channel condition of the corresponding link. In WANs, due to time-varying and location-dependent channel condition of each wireless link, not only the performance of each session but also the capacity of the wireless link depends on the scheduling policy. This is the one of the most distinguishing features of the wireless link compared with the wireline link in which the scheduling policy affects on only the performance of each session due to a fixed capacity. By using opportunistic scheduling in which sessions are scheduled according to their achievable instantaneous data rate in each time-slot, we can improve the capacity of the wireless link, i.e., the capacity of the system. Opportunistic scheduling is one of the most active research areas in wireless networks, e.g., [5], [6], [7], [8], [9]. However, they considered only a single hop WAN ignoring end-toend paths of sessions.

Recently, cross-layer optimization for the integrated wireless and wireline networks are studied in [10], [11]. Although a joint congestion control and MAC problem is considered in [10], the authors did not consider timevarying wireless channel condition and AMC at the physical layer. In [11], a joint congestion control and scheduling problem is considered. However, they formulated the optimization problem and implemented the algorithm for the network with a single WAN considering only the downlink of the WAN. In addition, the scheduling algorithm in [11] is implemented indirectly relying on the gradient algorithm in [5], [7]. Due to this reason, their algorithm includes imbedded iterations for scheduling that must converge within an iteration for link price update.

The problem in this paper is similar to that in [11]. However, there are several critical differences between them. Compared with [11], in this paper, we formulate the problem for the general network topology. In addition, we will use a unified dual based method with a stochastic sub-gradient algorithm without relying on the gradient algorithm, which results in no imbedded iterations within an iteration. This can be achieved by explicitly modeling time-varying wireless channel and appropriately using the partial dual relaxation technique in this paper. Usually, the algorithm with imbedded iterations may need more convergence time than the algorithm without imbedded iterations, since it needs enough time in each outer iteration for the inner iterations to be converged. The paper is organized as follows. In Section II, we introduce the system model. In Section III, we formulate our problem and present the joint flow control and scheduling algorithm. We provide numerical results in Section IV and conclude in Section V.

\section{SySTEM MODEL}

We consider a communication network that consists of a wireline $\mathrm{CN}$, set $A$ of WANs, and set $S$ of sessions, as in Fig. 1. We consider the end-to-end communication of each session $s$, whose end-to-end path consists of a subset $L(s)$ of links. We assume that the end-to-end path for each session are pre-determined. Determining the end-toend path for each session is beyond the scope of this paper. The first or/and last link of each session can be a wireless link in a WAN and other links consists of wireline links in the CN. Each session $s$ has its utility function $U_{s}\left(x_{s}\right)$, which is a function of its average end-to-end data rate $x_{s}$. We assume that each session $s$ has its minimum data rate requirement as its QoS requirement, i.e.,

$$
x_{s} \geq x_{s}^{\min } \text {. }
$$

The CN consists of set $L^{C}$ of wireline links. Each wireline link $l$ in the $\mathrm{CN}$ has a fixed capacity $C_{l}$. We define $L^{C}(s), s \in S$ as a subset of wireline links in the $\mathrm{CN}$ that session $s$ uses. We also define $S(l), l \in L^{C}$ as a set of sessions that use wireline link $l$ in the $\mathrm{CN}$. Hence, we have the following constraints for the capacity of each wireline link $l$ in the $\mathrm{CN}$.

$$
\sum_{s \in S(l)} x_{s} \leq C_{l}, \forall l \in L^{C} .
$$

Each WAN $a$ consists of a BS (or an AP) and set $S(a)$ of sessions. Sessions in each WAN is further divided into two subsets $S^{u}(a)$ and $S^{d}(a)$, where $S^{u}(a)$ is a set of sessions that use the uplink of WAN $a \in A$ and $S^{d}(a)$ is a set of sessions that use the downlink of WAN $a$. We define $a^{u}(s)$ as the WAN whose uplink is used by session $s$ and $a^{d}(s)$ as the WAN whose downlink is used by session $s$. The BS of each WAN is directly connected with the $\mathrm{CN}$ through a wireline link. Sessions in a WAN can be connected to the $\mathrm{CN}$ only through its $\mathrm{BS}$. We assume that frequency division duplexing is used for uplink and downlink communications in each WAN. Hence, we can deal with uplink and downlink problems in a WAN independently. We assume that each link in a WAN is time-slotted and a TDMA type of multiple access scheme is used for each of uplink and downlink. Hence, only one session can be scheduled in each time-slot for 
each of uplink and downlink. We assume that transmission power of BSs and users is fixed.

In contrary to a wireline link in the $\mathrm{CN}$ that has a fixed capacity, the capacity of a wireless link in a WAN depends on its scheduling policy due to the time-varying channel condition of each session. Hence, in this paper, we consider an opportunistic scheduling scheme in which the scheduling decision in each time-slot is based on the channel condition of each session. To this end, we model the time-varying channel condition of each session as a stationary stochastic process. In a time-slot, each of uplink and downlink of a WAN is assumed to be in one of several possible link states, in which each link state represents each of all possible combinations of the channel sates of all sessions that use the corresponding link. Hence, we represent the composite channel state of all sessions in a link of a WAN by using the corresponding link state. We define $L S^{u}(a)$ and $L S^{d}(a)$ as set of uplink states and set of downlink states of WAN $a$, respectively. We denote the probability that the uplink of WAN $a$ is in state $t$ as $\pi_{a}^{u}(t)$, $t \in L S^{u}(a)$ and the probability that its downlink is in state $t$ as $\pi_{a}^{d}(t), t \in L S^{d}(a)$.

In this paper, we adopt a probabilistic scheduling policy in which the BS in WAN $a$ randomly selects sessions $s^{u}$ and $s^{d}$ with probabilities $p_{a, s^{u}}^{u}\left(t^{u}\right)$ and $p_{a, s^{d}}^{d}\left(t^{d}\right)$ in a timeslot when the states of its uplink and downlink are $t^{u}$ and $t^{d}$, respectively. Hence, we have the following constraints for the scheduling in each link state of each of uplink and downlink in WAN $a$ :

$$
\begin{aligned}
Q_{a}^{u}(t)= & \left\{\bar{p}_{a}^{u}(t) \mid \sum_{s \in S^{u}(a)} p_{a, s}^{u}(t) \leq 1,\right. \\
& \left.0 \leq p_{a, s}^{u}(t) \leq 1, \forall s \in S^{u}(a)\right\},
\end{aligned}
$$

where $\bar{p}_{a}^{u}(t)=\left(p_{a, s}^{u}(t)\right)_{s \in S^{u}(a)}$, and

$$
\begin{aligned}
Q_{a}^{d}(t)= & \left\{\bar{p}_{a}^{d}(t) \mid \sum_{s \in S^{d}(a)} p_{a, s}^{d}(t) \leq 1,\right. \\
& \left.0 \leq p_{a, s}^{d}(t) \leq 1, \forall s \in S^{d}(a)\right\},
\end{aligned}
$$

where $\bar{p}_{a}^{d}(t)=\left(p_{a, s}^{d}(t)\right)_{s \in S^{d}(a)}$.

Since we assume that the transmission power of each of uplink and downlink is fixed, we can assume that the data rate of a session in a WAN depends only on its channel condition, i.e., the state of its link in the WAN. We define $r_{a, s}^{u}(t)$ as the achievable instantaneous data rate of session $s$ that uses the uplink of WAN $a$ when its uplink state is $t$. In a similar way, we define $r_{a, s}^{d}(t)$ as the achievable instantaneous data rate of session $s$ that uses the downlink of WAN $a$ when its downlink state is $t$. Hence, the achievable average data rate of session $s$ that uses the uplink of WAN $a$ is obtained as

$$
\sum_{t \in L S^{u}(a)} \pi_{a}^{u}(t) p_{a, s}^{u}(t) r_{a, s}^{u}(t)
$$

In addition, we have the following constraint for session $s$ that uses the uplink of WAN $a$ :

$$
x_{s} \leq \sum_{t \in L S^{u}(a)} \pi_{a}^{u}(t) p_{a, s}^{u}(t) r_{a, s}^{u}(t)
$$

which implies that the average data rate of a session should be less than or equal to the achievable average data rate of the wireless link that it uses. In a similar way, we have the following constraint for its downlink:

$$
x_{s} \leq \sum_{t \in L S^{d}(a)} \pi_{a}^{d}(t) p_{a, s}^{d}(t) r_{a, s}^{d}(t) .
$$

\section{Optimization Problem And Algorithm}

The goal of this paper is obtaining optimal data rate and scheduling probabilities of each session that maximizes network utility that is defined as the sum of all sessions' utilities. Hence, the problem with our objective and constraints that are derived in the previous section is formulated as

$$
\begin{aligned}
& \operatorname{maximize}_{\bar{p}, \bar{x}} \sum_{s \in S} U_{s}\left(x_{s}\right) \\
& \text { subject to } \\
& \sum_{s \in S(l)} x_{s} \leq C_{l}, \forall l \in L^{C}, \\
& x_{s} \leq \sum_{t \in L S^{u}(a)} \pi_{a}^{u}(t) p_{a, s}^{u}(t) r_{a, s}^{u}(t), \forall s \in S^{u}(a), \forall a \in A \\
& x_{s} \leq \sum_{t \in L S^{d}(a)} \pi_{a}^{d}(t) p_{a, s}^{d}(t) r_{a, s}^{d}(t), \forall s \in S^{d}(a), \forall a \in A \\
& x_{s} \geq x_{s}^{\min }, \forall s \in S \text {, } \\
& \bar{p}_{a}^{u}(t) \in Q_{a}^{u}(t), \forall t \in L S^{u}(a), \forall a \in A, \\
& \bar{p}_{a}^{d}(t) \in Q_{a}^{d}(t), \forall t \in L S^{d}(a), \forall a \in A \text {, }
\end{aligned}
$$

where $\bar{x}=\left(x_{s}\right)_{s \in S}, \quad \bar{p}=\left(\bar{p}_{a}^{u}, \bar{p}_{a}^{d}\right)_{a \in A}, \bar{p}_{a}^{u}=$ $\left(\bar{p}_{a}^{u}(t)\right)_{t \in L S^{u}(a)}, \quad \bar{p}_{a}^{d}=\left(\bar{p}_{a}^{d}(t)\right)_{t \in L S^{d}(a)}, \quad \bar{p}_{a}^{u}(t)=$ $\left(p_{a, s}^{u}(t)\right)_{s \in S^{u}(a)}$, and $\bar{p}_{a}^{d}(t)=\left(p_{a, s}^{d}(t)\right)_{s \in S^{d}(a)}$.

Although this problem is a convex optimization problem, in practice, it is not easy to have a prior information on link states, i.e., channel states of all wireless links. Hence, we need an algorithm that does not require such a priori information, and thus we will solve this problem by using a dual approach and a stochastic sub-gradient algorithm.

We first define the Lagrangian function associated with problem (1) as

$$
\begin{aligned}
& L(\bar{x}, \bar{p}, \bar{\lambda}, \bar{\mu})=\sum_{s \in S} U_{s}\left(x_{s}\right)+\sum_{l \in L^{C}} \lambda_{l}\left(C_{l}-\sum_{s \in S(l)} x_{s}\right) \\
& +\sum_{a \in A} \sum_{s \in S^{u}(a)} \mu_{a, s}^{u}\left(\sum_{t \in L S^{u}(a)} \pi_{a}^{u}(t) p_{a, s}^{u}(t) r_{a, s}^{u}(t)-x_{s}\right) \\
& +\sum_{a \in A} \sum_{s \in S^{d}(a)} \mu_{a, s}^{d}\left(\sum_{t \in L S^{d}(a)} \pi_{a}^{d}(t) p_{a, s}^{d}(t) r_{a, s}^{d}(t)-x_{s}\right)
\end{aligned}
$$




$$
\begin{aligned}
& =\sum_{s \in S}\left[U_{s}\left(x_{s}\right)-x_{s}\left(\sum_{l \in L^{C}(s)} \lambda_{l}+\mu_{a, s}^{u}+\mu_{a, s}^{d}\right)\right] \\
& +\sum_{l \in L^{C}} \lambda_{l} C_{l} \\
& +\sum_{a \in A} \sum_{s \in S^{u}(a)} \mu_{a, s}^{u}\left(\sum_{t \in L S^{u}(a)} \pi_{a}^{u}(t) p_{a, s}^{u}(t) r_{a, s}^{u}(t)\right) \\
& +\sum_{a \in A} \sum_{s \in S^{d}(a)} \mu_{a, s}^{d}\left(\sum_{t \in L S^{d}(a)} \pi_{a}^{d}(t) p_{a, s}^{d}(t) r_{a, s}^{d}(t)\right)
\end{aligned}
$$

where $\bar{\lambda}=\left(\lambda_{l}\right)_{l \in L^{C}}, \quad \bar{\mu}=\left(\bar{\mu}_{a}^{u}, \bar{\mu}_{a}^{d}\right)_{a \in A}, \quad \bar{\mu}_{a}^{u}=$ $\left(\mu_{a, s}^{u}\right)_{s \in S^{u}(a)}$, and $\bar{\mu}_{a}^{d}=\left(\mu_{a, s}^{d}\right)_{s \in S^{d}(a)}$. Note that here we do not relax last three constraints in (1). We now define the dual objective function $Q(\bar{\lambda}, \bar{\mu})$ as:

$$
\begin{aligned}
& Q(\bar{\lambda}, \bar{\mu})= \\
& \max _{\bar{x}>\bar{x}^{\text {min }}} \\
& \bar{p}^{u}(t) \in Q^{u}(t), \forall t \in L S^{u}(a), \forall a \in A \\
& \bar{p}_{a}^{d}(t) \in Q_{a}^{d}(t), \forall t \in L S^{d}(a), \forall a \in A
\end{aligned}
$$

where $\bar{x}^{\text {min }}=\left(x_{s}^{\text {min }}\right)_{s \in S}$.

Now, the dual problem is defined as

$$
\operatorname{minimize}_{\bar{\lambda} \geq \overline{0}, \bar{\mu} \geq \overline{0}} Q(\bar{\lambda}, \bar{\mu}) .
$$

To solve the dual problem in (3), we first consider the optimization problem in (2). For given $\bar{\lambda}$ and $\bar{\mu}$, the solutions of this problem $\bar{x}(\bar{\lambda}, \bar{\mu})=\left(x_{s}(\bar{\lambda}, \bar{\mu})\right)_{s \in S}$ and $\bar{p}(\bar{\mu})=\left(p_{a}^{u}(\bar{\mu}), p_{a}^{d}(\bar{\mu})\right)_{a \in A}$ are obtained as

$$
\begin{aligned}
& x_{s}(\bar{\lambda}, \bar{\mu})=\underset{x_{s} \geq x_{s}^{\min }}{\operatorname{argmax}}\left\{U_{s}\left(x_{s}\right)-x_{s}\left(\sum_{l \in L(s)} \lambda_{l}\right.\right. \\
& \left.\left.+\mu_{\left.a^{u}(s), s\right)}^{u}+\mu_{a^{d}(s), s}^{d}\right)\right\}, \forall s \in S . \\
& \bar{p}_{a}^{u}(\bar{\mu})=\underset{\bar{p}_{a}^{u}(t) \in Q_{a}^{u}(t), \forall t \in L S^{u}(a)}{\operatorname{argmax}}\left\{\sum_{t \in L S^{u}(a)} \pi_{a}^{u}(t)\right. \\
& \left.\sum_{s \in S^{u}(a)} \mu_{a, s}^{u} p_{a, s}^{u}(t) r_{a, s}^{u}(t)\right\}, \forall a \in A
\end{aligned}
$$

and

$$
\begin{gathered}
\bar{p}_{a}^{d}(\bar{\mu})=\underset{\bar{p}_{a}^{d}(t) \in Q_{a}^{d}(t), \forall t \in L S^{d}(a)}{\operatorname{argmax}}\left\{\sum_{t \in L S^{d}(a)} \pi_{a}^{d}(t)\right. \\
\left.\sum_{s \in S^{d}(a)} \mu_{a, s}^{d} p_{a, s}^{d}(t) r_{a, s}^{d}(t)\right\}, \forall a \in A .
\end{gathered}
$$

Since problems in (5) and (6) are separable in $t$, they can be further decomposed as

$$
\begin{gathered}
\bar{p}_{a}^{u}(\bar{\mu}, t)=\underset{\bar{p}_{a}^{u}(t) \in Q_{a}^{u}(t)}{\operatorname{argmax}} \sum_{s \in S^{u}(a)} \mu_{a, s}^{u} p_{a, s}^{u}(t) r_{a, s}^{u}(t), \\
\forall t \in L S^{u}(a), \forall a \in A
\end{gathered}
$$

and

$$
\begin{gathered}
\bar{p}_{a}^{d}(\bar{\mu}, t)=\underset{\bar{p}_{a}^{d}(t) \in Q_{a}^{d}(t)}{\operatorname{argmax}} \sum_{s \in S^{d}(a)} \mu_{a, s}^{d} p_{a, s}^{d}(t) r_{a, s}^{d}(t), \\
\forall t \in L S^{d}(a), \forall a \in A,
\end{gathered}
$$

where $\bar{p}_{a}^{u}(\bar{\mu}, t)=\left(\bar{p}_{a}^{u}(\bar{\mu}, t)\right)_{t \in L S^{u}(a)}$ and $\bar{p}_{a}^{d}(\bar{\mu})=$ $\left(\bar{p}_{a}^{d}(\bar{\mu}, t)\right)_{t \in L S^{d}(a)}$. Hence, we can solve problems in (7) and (8) (i.e., problems in (5) and (6)) even without knowledge of probability distributions of link states. The solutions of problems in (7) and (8) are obtained as

$$
\begin{aligned}
& p_{a, s}^{u}(\bar{\mu}, t)=\left\{\begin{array}{ll}
1, & \text { if } s=\underset{i \in S^{u}(a)}{\operatorname{argmax}} \mu_{a, i}^{u} r_{a, i}^{u}(t) \\
0, & \text { otherwise }
\end{array},\right. \\
& \forall t \in L S^{u}(a), \forall a \in A
\end{aligned}
$$

and

$$
\begin{aligned}
& p_{a, s}^{d}(\bar{\mu}, t)=\left\{\begin{array}{ll}
1, & \text { if } s=\underset{i \in S^{d}(a)}{\operatorname{argmax}} \mu_{a, i}^{d} r_{a, i}^{d}(t) \\
0, & \text { otherwise }
\end{array},\right. \\
& \forall t \in L S^{u}(a), \forall a \in A \text {. }
\end{aligned}
$$

We now consider the dual problem in (3) and it can be solved by using the following stochastic subgradient algorithm.

$$
\begin{aligned}
\lambda_{l}(n+1)= & {\left[\lambda_{l}^{n}-\alpha(n)\left(C_{l}\right.\right.} \\
& \left.\left.-\sum_{s \in S(l)} x_{s}(\bar{\lambda}(n), \bar{\mu}(n))\right)\right]^{+}, \forall l \in L^{C}, \\
\mu_{a, s}^{u}(n+1) & =\left[\mu_{a, s}^{u}(n)-\alpha(n)\left(p_{a, s}^{u}(\bar{\mu}(n), t(n)) r_{a, s}^{u}(t(n))\right.\right. \\
& \left.\left.-x_{s}(\bar{\lambda}(n), \bar{\mu}(n))\right)\right]^{+}, \forall s \in S^{u}(a), \forall a \in A,
\end{aligned}
$$

and

$$
\begin{aligned}
\mu_{a, s}^{d}(n+1) & =\left[\mu_{a, s}^{d}(n)-\alpha(n)\left(p_{a, s}^{d}(\bar{\mu}(n), t(n)) r_{a, s}^{d}(t(n))\right.\right. \\
& \left.\left.-x_{s}(\bar{\lambda}(n), \bar{\mu}(n))\right)\right]^{+}, \forall s \in S^{d}(a), \forall a \in A,
\end{aligned}
$$

where $\alpha(n)$ is a step size, $x_{s}(\bar{\lambda}(n), \bar{\mu}(n))$ is a solution of the problem in (4), $p_{a, s}^{u}(\bar{\mu}(n), t(n))$ and $p_{a, s}^{d}(\bar{\mu}(n), t(n))$ are solutions of problems in (7) and (8) with $\bar{\lambda}(n)$ and $\bar{\mu}(n)$ at time-slot $n$, and $t(n)$ is the link state at time-slot $n$. Hence, the problem in (1) can be solved iteratively and in a distributed way involving all four layers in the network layering architecture and all entities in the network such as the source of each session, each link in the $\mathrm{CN}$, and WANs as follows. At each time-slot $n$, the below updates should be done in a distributed way.

End-to-end data rate update at the source of each session 
The source of session $s$ updates its end-to-end data rate by solving

$$
\begin{aligned}
x_{s}(n) & =\underset{x_{s} \geq x_{s}^{\min }}{\operatorname{argmax}}\left[U_{s}\left(x_{s}\right)-x_{s}\left(\sum_{l \in L(s, p)} \lambda_{l}(n)\right.\right. \\
& \left.\left.+\mu_{a^{u}(s), s}^{u}(n)+\mu_{a^{d}(s), s}^{d}(n)\right)\right], \forall s \in S .
\end{aligned}
$$

Here, dual variables $\lambda$ 's and $\mu$ 's are delivered from links that the path of the session are using. Note that the above problem is a convex optimization problem with simple nonnegativity constraints for variables. Hence, it can be easily solved by using a standard optimization algorithm. Dual variable update at each link in the $\mathrm{CN}$

Each link $l$ in the $\mathrm{CN}$ updates its dual variable by solving

$$
\lambda_{l}(n+1)=\left[\lambda_{l}^{n}-\alpha(n)\left(C_{l}-\sum_{s \in S(l)} x_{s}(n)\right)\right]^{+},
$$

Here, the aggregate average data rate $\sum_{s \in S(l)} x_{s}(n)$ can be obtained by measuring it or by explicitly notification from the sources of sessions that are using the link.

\section{Opportunistic scheduling at each WAN}

The BS in each WAN $a$ schedules sessions for each of its uplink and downlink with probabilities $\bar{p}_{n}^{u}(a)$ and $\bar{p}_{n}^{d}(a)$ by solving

$p_{a, s}^{u}(n)=\left\{\begin{array}{ll}1, & \text { if } s=\underset{i \in S^{u}(a)}{\operatorname{argmax}} \mu_{a, i}^{u}(n) r_{a, i}^{u}(n) \\ 0, & \text { otherwise }\end{array}, \forall a \in A\right.$ and

$p_{a, s}^{d}(n)=\left\{\begin{array}{ll}1, & \text { if } s=\underset{i \in S^{d}(a)}{\operatorname{argmax}} \mu_{a, i}^{d}(n) r_{a, i}^{d}(n) \\ 0, & \text { otherwise }\end{array}, \forall a \in A\right.$.

Here, the achievable instantaneous data rates of its sessions for its uplink and downlink, $r_{a, i}^{u}(n)$ 's and $r_{a, i}^{d}(n)$ 's can be calculated by the BS by measuring the channel state (e.g., SINR) of each of its sessions. In addition, dual variables $\mu$ 's are calculated by the BS

\section{Dual variable update at each link in the WAN}

The BS in each WAN $a$ updates dual variables for its sessions for each of its uplink and downlink by solving

$$
\begin{aligned}
\mu_{a, s}^{u}(n+1) & =\left[\mu_{a, s}^{u}(n)-\alpha(n)\left(p_{a, s}^{u}(n) r_{a, s}^{u}(n)\right.\right. \\
& \left.\left.-x_{s}(n)\right)\right]^{+}, \forall s \in S^{u}(a), \forall a \in A
\end{aligned}
$$

and

$$
\begin{aligned}
\mu_{a, s}^{d}(n+1) & =\left[\mu_{a, s}^{d}(n)-\alpha(n)\left(p_{a, s}^{d}(n) r_{a, s}^{d}(n)\right.\right. \\
& \left.\left.-x_{s}(n)\right)\right]^{+}, \forall s \in S^{d}(a), \forall a \in A .
\end{aligned}
$$

Here, the achievable instantaneous data rates of sources $r_{s}^{u}(n)$ 's and $r_{s}^{d}(n)$ 's, and schedule probabilities $p_{a, s}^{u}(n)$ 's and $p_{a, s}^{u}(n)$ 's are calculated by the BS. In addition, the average transmitting data rate of its sessions, $x_{s}(n)$ can be

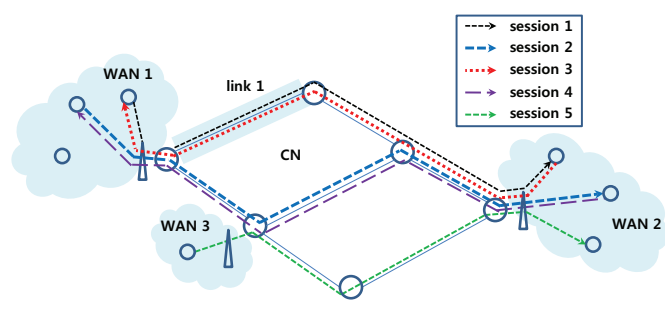

Fig. 2. The topology of the network in our simulations.

obtained by measuring it or by explicitly notification from the sources of sessions that are using the corresponding link of WAN $a$.

\section{Simulation Results}

In this section, we provide numerical results for our algorithm. We consider a network in Fig. 2 that consists of three WANs that are connected to a wireline $\mathrm{CN}$. We set the capacity of each wireline link in the $\mathrm{CN}$ to be 10 Mbps except link 1, whose capacity is set to be 3 Mbps. This implies that link 1 in the $\mathrm{CN}$ could be a bottleneck link. In each WAN, the bandwidth of each of uplink and downlink is set to be $1 \mathrm{MHz}$. the achievable data rate of each session is assumed to follow the Shannon capacity. We set the average SNR of all wireless links to be $20 \mathrm{~dB}$, except session 1's uplink in WAN 1, whose average SNR is set to be $0 \mathrm{~dB}$. This implies that the source mobile node of session in WAN 1 has a relatively worse channel condition than other mobile nodes. We model the stochastically varying channel condition of each wireless link by using Rayleigh fading. We assume that each session $s$ has a log utility function, i.e., $U_{s}\left(x_{s}\right)=\log \left(x_{s}\right)$.

To show the appropriateness of our approach, i.e., joint optimization for both WANs and wireline $\mathrm{CN}$, we also provide results that are obtained by the conventional approaches, i.e., the optimal flow control algorithm considering only the wireline $\mathrm{CN}$ and the optimal opportunistic scheduling algorithm considering only a single WAN

In Fig. 3(a), we provide average data rates of session 1 that are offered by its uplink in WAN 1 and downlink in WAN 2 through the separate opportunistic scheduling algorithm at each of WANs without considering the wireline $\mathrm{CN}$. We also provide its average data rate that is calculated at its source node through the flow control algorithm considering only the wireline $\mathrm{CN}$. As shown in this figure, even though those three data rates correspond to the same session, they converge to a different value to each other. This implies that the actual average data rate of session 1 cannot exceed the minimum of those three values, i.e, the average data rate at the uplink of WAN 1, while resources that are allocated at other networks, i.e., $\mathrm{CN}$ and WAN 2, are wasted.

In Fig. 3(b), we also provide those three data rates of session 1 that are obtained through our joint algorithm. As this figure shows, all three data rates converge to 


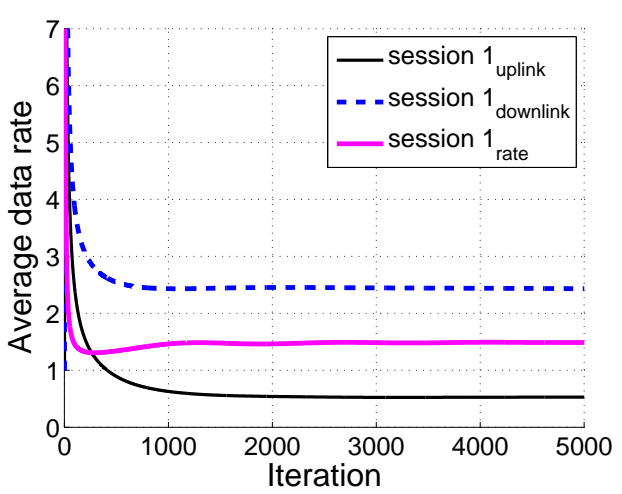

(a) Conventional separated and layered approach.

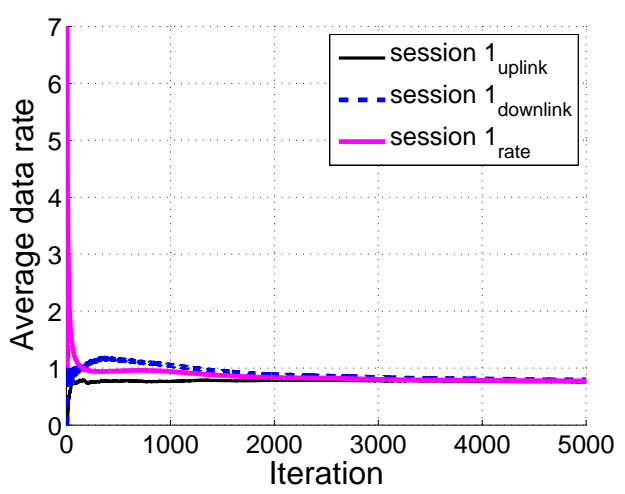

(b) Our joint and cross-layered approach.

Fig. 3. The comparison of the data rates of session 1

the same value. As mentioned above, for session 1, the uplink in WAN 1 is a bottleneck link. Hence, the $\mathrm{CN}$ and the downlink of WAN 2 only have to allocate resources just enough to support the average data rate offered to session 1 offered by the uplink in WAN 1 and remaining resources can be allocated to other sessions to improve system efficiency.

In Fig. 4, we compare the achieved utility of each session obtained through our joint approach and that obtained through the conventional separated approach. We also compare the total network utilities obtained by those two approaches. As we have shown in Fig. 3, in the separated approach, even for the same session, there might exist mismatches among data rates in different networks, which causes the waste of network resources. On the other hand, in our joint approach, since all different networks are jointly optimized, network resources can be utilized more efficiently. Hence, as shown in this figure, our joint approach provides higher utilities than the conventional separated approach.

\section{CONCLUSION}

In this paper, we study a joint optimization problem with wireline core network and wireless access networks. We formulate and solve the optimization problem considering

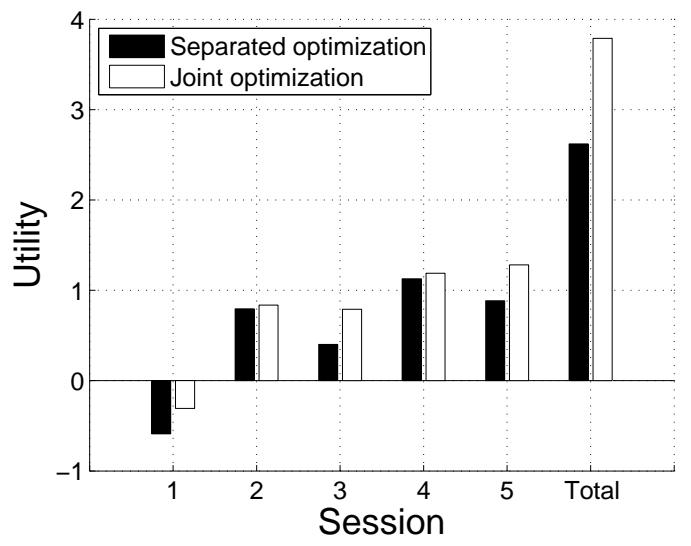

Fig. 4. The comparison of the achieved utilities.

application, transport, MAC, and physical layers in the network layering architecture, resulting in a jointly and cross-layer optimized utility-based flow control and opportunistic scheduling for integrated wireline and wireless network. The algorithm can be implemented in a distributed way in each network entity and each layer with minimal information exchanges. Numerical results show that our joint approach provides a better performance than the conventional separated approach.

\section{REFERENCES}

[1] F. Kelly, A. Maulloo, and D. Tan, "Rate control in communication networks: Shadow prices, proportional fairness and stability," Journal of the Operational Research Society, vol. 49, no. 3, pp. 237-252, Mar. 1998.

[2] S. H. Low and D. E. Lapsley, "Optimization flow control - I: Basic algorithm and convergence," IEEE/ACM Transactions on Networking, vol. 7, no. 6, pp. 861-874, Dec. 1999.

[3] J.-W. Lee, R. Mazumdar, and N. B. Shroff, "Non-convex optimization and rate control for multi-class services in the internet," IEEE/ACM Transactions on Networking, vol. 13, no. 4, pp. 827840, Aug. 2005.

[4] B.-G. Kim and J.-W. Lee, "Utility-based end-to-end flow control for services with time-varying rate requirements," in IEEE CCNC, Jan. 2009.

[5] R. Agrawal and V. Subramanian, "Optimality of certain channel aware scheduling policies," in Allerton Conference on Communication, Control and Computing, 2002.

[6] X. Liu, E. Chong, and N. Shroff, "A framework for opportunistic scheduling in wireless networks," vol. 41, pp. 451-474, Mar. 2003.

[7] A. L. Stolyar, "On the asymptotic optimality of the gradient scheduling algorithm for multiuser throughput allocation," Operation Research, vol. 53, no. 1, pp. 12-25, Jan. 2005.

[8] J.-W. Lee, R. R. Mazumdar, and N. B. Shroff, "Opportunistic power scheduling for dynamic multi-server wireless systems," IEEE Transactions on Wireless Communications, vol. 5, no. 6, pp. 15061515, Jun. 2006.

[9] J. Huang, V. Subramanian, R. Agrawal, and R. Berry, "Downlink scheduling and resource alloction for OFDM systems," IEEE Transactions on Wireless Communications, vol. 8, no. 1, pp. 1-9, Jan. 2009.

[10] X. Wang, K. Kar, and S. H. Low, "End-to-end fair rate optimization in wired-cum wireless networks," Ad-Hoc Netowrks, vol. 7, no. 3, pp. 473-485, May 2009.

[11] Y. Yu and G. B. Giannakis, "Joint congestion control and ofdma scheduling for hybrid wireline-wireless networks," in IEEE INFOCOM, 2007, pp. 973-981. 